\title{
Archipel
}

ARCHIPEL Études interdisciplinaires sur le monde insulindien

$88 \mid 2014$

Varia

\section{Flussaufwärts, Die Borneo Sammlung Hilde May}

\section{Antonio Guerreiro}

\section{OpenEdition}

Journals

Édition électronique

URL : http://journals.openedition.org/archipel/537

DOI : 10.4000/archipel.537

ISSN : 2104-3655

\section{Éditeur}

Association Archipel

\section{Édition imprimée}

Date de publication : 10 octobre 2014

Pagination : 217-221

ISBN : 978-2-910513-71-9

ISSN : 0044-8613

\section{Référence électronique}

Antonio Guerreiro, « Flussaufwärts, Die Borneo Sammlung Hilde May », Archipel [En ligne], 88 | 2014, mis en ligne le 10 septembre 2017, consulté le 25 septembre 2020. URL : http://

journals.openedition.org/archipel/537 ; DOI : https://doi.org/10.4000/archipel.537 
growth of George Town from the end of $18^{\text {th }}$ century up to the mid- $20^{\text {th }}$ century, is the first of its kind. However the interest for local figures started much earlier when, in 1986, a book entitled Historical Personalities of Penang was published by The Historical Personalities of Penang Committee under the Auspices of Penang Festival'86, and an exhibition based on this publication inaugurated. This latter volume also included community and political leaders, civil servants, academicians, religious teachers and artists, and among them, a few prominent women, but the biographical sketches were not referenced.

The present book highlights the life of merchants and business people from all communities, including the Acehnese, Arabs, Armenians, British, Chinese, Eurasians, Indians, Malays, Parsees, Scottish. It has been prepared with great care, thanks to the contribution of twenty authors. The entries are introduced by an overview of the historical development of the island since the coming of the Portuguese to Malacca (pp. 4-8). It appears clearly that the trader and business community has formed a major pillar since the late $18^{\text {th }}$ century of the city, when Penang was made an entrepôt.

Then the editors in a note on the biographies (200) explain how they proceeded. Each entry begins with the surname, given name (Chinese names in Hokkien dialect, followed by characters, and also in Mandarin, transcribed in pinyin), date of birth and death, profession, occupation, major role in the society. It ends with references in abbreviated form, while full references are given in a general bibliography including public records, archives and archival materials, newspapers and magazines (in English, Malay, and Chinese), unpublished theses, papers and manuscripts, personal communications, books and journal articles (in English, Malay and Chinese), and internet sources. The use of a symbol in the index allows the reader to know if a personality is listed in the main entries (pp. 12-178) or in the brief biographical sketches (24 altogether, pp. 179-191) that is those for whom the biodata are not yet sufficiently documented, but who merit inclusion in the history of Penang. If we consider that in 1810 , the Indians represented some $30 \%$ of the population, Chinese $36 \%$, Malays and Arabs $25 \%$ and that in 1860, the Chinese became the majority in Penang, one would not be surprised to notice that they are also the best represented among the biographies.

Although this dictionary is still far from being exhaustive, as the editors admit, it does great service to the historians who want to deepen their knowledge on these personalities and their part in the making of Malaya and even Southeast Asia.

Claudine Salmon

Stefan Dietrich \& Margareta Pavaloi (eds), Flussaufwärts, Die Borneo Sammlung Hilde May, Heidelberg : Völkerkundemuseum der J. \& E. von Portheim-Stiftung (vPST), 2013, 200 pages, 283 ill., bibliographie.

Preuve d'un regain d'intérêt pour les arts et les cultures de Bornéo depuis quelques années ${ }^{1}$, ce catalogue d'exposition présente une collection formant un ensemble représentatif

1. Il faut citer les expositions récentes sur l'art des sociétés dayak, notamment la statuaire et la sculpture « Patong », organisée en 2007 au Museo delle Culture de Lugano (Suisse), à partir de l'importante collection d'art tribal Brignoni, léguée au musée (Patong. La grande sculpture dei popoli del Borneo, Milan, Mazzota, 2008). Puis, dans le même établissement en 2012 une exposition centrée sur les chapeaux de soleil de la province de Kalimantan Centre (Sapuyung. Cappelli cerimoniali del Borneo, Milan, Mazotta, 2012). En France, il faut citer l'exposition «Dayak et Punan : Peuples de la forêt tropicale » au Musée d'Art et d'Archéologie de Laon dans l'Aisne en 2006 - sans catalogue ; plus 
de la culture matérielle dayak, ainsi que des objets de prestige acquis par ces derniers depuis plusieurs siècles (jarres et bols de céramique chinoise et d'Asie du Sud-Est (Siam), objets en bronze et laiton fabriqués par les Malais de Bornéo). Il s'agit de la collection de Madame Hilde May (1940-2005). Le catalogue, publié sous la direction de Stefan Dietrich et Margareta Pavaloi, accompagnait l'exposition éponyme qui s'est tenue du 3 mars 2013 au 6 juin 2014 au musée ethnologique de la Fondation J. \& E. von Portheim à Heidelberg. Le titre, «En amont de la rivière », fait référence à la vaste région du bassin du Mahakam, connue localement dans la province de Kalimantan Est sous le nom de Hulu Mahakam, « l'amont du Mahakam ». Elle englobe les kabupaten contemporains de Kutai Kertanegara, Kutai Barat, et Kutai Timur, dont les limites ont été définies à partir de l'ancien kabupaten Kutai au moment de la mise en place de l'autonomie régionale (otda) en 2000. Vient s'y ajouter en 2013 un nouveau kabupaten nommé Mahakam Hulu, découpé dans le territoire de Kutai Barat. Les pièces exposées viennent en grande majorité de cette aire complexe d'un point de vue ethnolinguistique. Mais elle peut se réduire à deux grands ensembles culturels : d'une part les populations benua'-bentian et tunjung, apparentées aux Luangan et Dusun plus au sud et au sud-ouest (dans l'aire de Pasir et sur les franges des provinces de Kalimantan Sud et de Kalimantan Tengah), d'autre part les Aoheng du Haut Mahakam et les Bahau Busang, Bahau Saa' et Modang, ces derniers venus des hauts plateaux du centre de l'île, établis plus au nord. Il faut y ajouter les quelques groupes punan qui vivent dans la même région.

Il n'est pas inutile de rappeler l'origine de cette importante collection. En mai 1977, Hilde May, accompagnant son mari, Bernhard May, économiste, nommé directeur du projet Transmigration Area Development (T.A.D.) ${ }^{2}$, arrive à Samarinda, la capitale provinciale de Kalimantan Est (Kaltim) où elle résidera plusieurs années jusqu'en 1985 - ensuite elle vivra à Jakarta. Hilde May commence rapidement à acquérir des pièces dayak, d'abord dans un petit magasin de la ville nommé « Toko Bali », qui vendait aussi des objets d'autres régions de l'archipel. Sans formation particulière dans ce domaine, elle se passionne pour l'artisanat et l'art des Dayak. Puis à partir de 1978 elle fera des expéditions dans l'intérieur du bassin du Mahakam, visitant les villages benua'-tunjung, kenyah, bahau, modang, et à chaque fois elle ramènera des pièces intéressantes. Même installée à Jakarta, elle continuera à faire des voyages à Kaltim, en pays dayak. Madame May précise qu'elle a surtout été en contact avec les habitants âgés de 30 à 50 ans qui parlaient l'indonésien à l'époque, ce qui n'était pas le cas de nombre de personnes plus âgées. C'est en 1979, qu'elle prendra, grâce à un ami commun, un premier contact avec le directeur du musée ethnologique de la Fondation von Portheim-Stiftung. Il insiste pour qu'elle recueille suffisamment de données sur le contexte culturel des objets, la « nature des choses ». Après des échanges avec le géographe spécialiste de Bornéo, Karl Helbig - décédé en 1991-, l'idée de déposer la collection dans un musée afin

récemment celle de l'espace Saint-Jacques à Saint-Quentin en mars-avril 2013 (« De la forêt tropicale aux mythes. Arts et cultures dayak de Bornéo »). Enfin, au Musée d'Anthropologie et d'Archéologie de l'Université de Cambridge de mai à août 2013, l'exposition « The Cultured Rainforest », centrée sur la vallée de la rivière Kelapang (hauts-plateaux kelabit, Sarawak), met en valeur les objets ethnographiques, en regard du paysage forestier et des monuments mégalithiques, dans le contexte de fouilles archéologiques menées dans la région de 2007 à 2011.

2. Le projet avait été mis en place dans le cadre de coopération germano-indonésienne (GTZ) avec le ministère de la transmigration de l'époque. Il s'agissait de définir des axes et des projets pilotes pour la construction des infrastructures et développer le potentiel économique de la province (routes, plantations, pêcheries, système de santé...). Il continuera ses activités jusqu'au début des années 1990, l'atelier de cartographie du T.A.D. étant ensuite rattaché au BAPPEDA (Daerah Tingkat I) à Samarinda. 
qu'elle soit accessible au plus grand nombre fait son chemin. Depuis 1983, grâce à la vaste bibliothèque de ce dernier, elle s'était plongée dans la littérature ethnographique sur Bornéo. Finalement, la collection sera déposée en juin 1992 au musée de la Fondation. Par la suite, pendant plus de vingt ans, elle complétera autant que possible la documentation des pièces, dans l'espoir que la collection soit exposée et qu'un catalogue raisonné soit publié à cette occasion. Le décès prématuré de Hilde May ne lui a pas permis de voir l'exposition, comme le rappelle Bernhard May dans un post-scriptum à la préface, qui est en fait un fragment d'un texte inachevé de son épouse sur l'histoire de la collection (p. 9-11). Finalement la collection Hilde May sera donnée au musée par B. May, et son soutien actif a rendu possible l'exposition et la publication.

Ce livre catalogue comprend un ensemble de six contributions, en sus de l'avant-propos des éditeurs (p. 5-6) et de la préface de Hilde May mentionnée plus haut. Le premier chapitre, rédigé par Stefan Dietrich à partir d'une étude approfondie de la littérature ethnographique, aborde de manière générale la région du Mahakam et les caractéristiques sociales et culturelles des ethnies dayak qui y sont établies (p. 14-92). Il examine l'organisation socio-politique, l'habitat en longue maison, l'essartage du riz (padi), ainsi que la représentation du monde, notamment à travers une analyse des mythes kayan et benua'-bentian (p. 67-77), ainsi que le rôle de l'adat, les différentes cérémonies et les objets ou constructions qui s'y rapportent (fête de la moisson du riz, danses masquées hudo', rites funéraires). Je note au passage une erreur à propos de l'ethnonyme " bahau », l'auteur confondant la distinction linguistique Bahau jaan et Bahau batè - jaan et batè signifiant « non » dans leurs dialectes —, avec la différenciation ethnique territoriale Bahau Busang et Bahau Saa', définissant les groupements respectivement de l'amont et de l'aval du Mahakam (p. 19). Les chapitres suivants sont centrés sur des catégories d'objets particuliers. Margareta Pavaloi a signé deux chapitres, le premier présente la ciselure et la sculpture sur bois à côté de la vannerie en rapport avec les représentations socio-religieuses (p.93-118). Le second étudie le textile, à partir des vêtements (jupes, vestes, coiffes et chapeaux), et les parures, dont les perles de verre, et celles réalisées en autres matériaux (métal, bois, pierre), les pendants et bouchons d'oreilles, les portebébés bahau et kenyah, ainsi que le tatouage avec ses instruments. Robert Bitsch traite de la métallurgie dayak. À partir des sources anciennes, il aborde la fonte et la forge des lames des fameux sabres mandau et d'autres armes et outils, sans oublier les superbes poignées ciselées des sabres ; il continue en décrivant l'équipement du guerrier (boucliers, coiffes, lances, sarbacanes...). Les deux derniers chapitres prennent pour objet, respectivement la céramique (Brigitte Borel, p. 173-186) et, autour du thème de la chique de bétel, les objets d'importation en bronze et en laiton, certains venus probablement de Brunei (bouilloires, plateaux, jarres...) et d'autres de Negara (nécessaires à bétel, boîtes, crachoirs...), un centre de la métallurgie du laiton dans le pays banjar à Kalimantan Sud (Pavaloi et Dietrich, p. 187-195). Les bols en céramique chinoise sont originaires du sud de la Chine (Fujian ou Guangdong). Ils datent de la fin de la dynastie Qing, $\mathrm{XIX}^{\mathrm{e}}-\mathrm{XX}^{\mathrm{e}}$ siècles, certaines jarres du Siam et de Chine étant plus anciennes, $\operatorname{circa} \mathrm{XVI}^{\mathrm{e}}$-XVII ${ }^{\mathrm{e}}$ siècles. Dans chacun des chapitres les auteurs contextualisent les objets selon leurs matériaux et leurs usages à partir des données de la littérature, les références bibliographiques étant placées en notes de fin de chapitre. Un exercice qui pourrait être rébarbatif mais il se lit facilement. L'accent n'est pas mis sur l'aspect technologique ou les matériaux, mais plutôt sur les descriptions intégrant les aspects esthétiques, historiques et culturels et les usages en rapport aux pièces de la collection. À ce propos, la qualité et le nombre des illustrations, aussi bien en noir et blanc qu'en couleur, est un point fort du livre. 
Il faut saluer le choix des éditeurs qui ont décidé d'alterner les photographies contemporaines de Bernhard et Hilde May (26) et les images d'archives (46) de la fin du XIX / début XX $\mathrm{XX}^{\mathrm{e}}$ siècle, toutes très bien imprimées (d'après Hose, Ch. and MacDougall, M., The Pagan Tribes of Borneo, 1912 et Nieuwenhuis, A.W. Quer Durch Borneo, 1904-1907). Les photographies des hommes et de leurs productions matérielles in situ s'insèrent harmonieusement entre les illustrations en couleur des objets de la collection prises au musée (211).

Le livre complète heureusement les publications existantes sur l'art et l'artisanat des sociétés dayak, plus particulièrement pour la région du Mahakam ${ }^{3}$. À côté des objets anciens, « importés » dans les villages dayak (céramiques, perles de verre, objets en bronze et laiton), la collection Hilde May comporte surtout des objets relativement récents, datant des années 1930 à 1980, et quelques pièces plus anciennes, datant des années 1890-1920. Dans son ensemble, la collection témoigne de la maîtrise des artisans dayak qui excellent dans tous les domaines de l'art décoratif, notamment le travail du bois et de l'os. Je relève que la majorité des pièces illustrées dans l'ouvrage provient de l'aval des grands rapides du Mahakam (hilir kiham). Hilde May avait su équilibrer ses collectes entre les objets cérémoniels et de prestige et ceux de la vie quotidienne. Mais il faut souligner à regret que de rares " curios », fabriqués localement pour la vente dans un cadre touristique ou non ${ }^{4}$, se sont glissés aux côtés des objets utilisés par les populations. Il aurait été judicieux de ne pas les intégrer à côté des autres pièces. Ce point laisse également ouvert la question de la sélection des objets par les organisateurs de l'exposition, le nombre de pièces ou lots de la collection Hilde May déposés au musée n'étant pas précisé dans le catalogue : cette dernière a-t-elle laissé des notes à propos de ses collectes (provenances, conditions d'acquisition, techniques...) ?

On note également quelques approximations quant à l'attribution ethnique et / ou la fonction de certaines pièces : par exemple le $\mathrm{n}^{\circ} 108$ est un décor de proue de pirogue bahau, pas d'une extrémité d'ossuaire benua' (p. 86) ; les chapeaux d'hommes, illustration $\mathrm{n}^{\circ} 179$, sont respectivement aoheng / kayaan (gauche) et bahau busang (droite) sans rapport avec les Kenyah de l'Apo Kayan (p. 137) ; les $n^{\circ} 222$ et $n^{\circ} 236$, des boucles d'oreilles en ivoire de calao casqué (Rhinoplax vigil) sont bien de style kenyah et non pas bahau (p. 158, 160). La disparition prématurée de la collectrice avant la finalisation du projet d'exposition n'y est sans doute pas étrangère.

3. Guerreiro, A. et Sellato, B., De la forêt tropicale aux mythes. Arts et cultures dayak de Bornéo, municipalité de Saint-Quentin, 2013 ; Juynboll, H.H., Catalogus van's Rijks ethnographisch Museum. Borneo, deel I (Borneo erste gedeelte), Borneo, deel II (Borneo tweede gedeelte), Leiden, E.J. Brill, 1910 ; Nieuwenhuis, A.W., Quer Durch Borneo, Leiden, E. J. Brill, vol. I, (1904), vol II (1907). Sellato, B., Hornbill and Dragon / Naga dan Burung Enggang, Jakarta / Kuala Lumpur, Elf Aquitaine, 1989.

4. Parmi ces pièces, qui visiblement sont récentes, je remarque inter alia des masques de style hudo' « hybride», les $n^{\text {os }} 61$ et 66 ; une « sculpture » janus, $n^{\circ} 82$; un collier « à amulettes », $n^{\circ} 102 ;$ le $n^{\circ} 116$, un carquois en bambou pour fléchettes de sarbacane ; le $\mathrm{n}^{\circ} 125$, un plat en bois. Par ailleurs les cinq figurines en position accroupie, sculptées en pierre ( $\left.{ }^{\text {os }} 12-16, p .23\right)$, sont problématiques quant à leur fonction et à leur attribution ethnique. Ces pièces ne sont pas présentées en tant qu'objets témoins du changement culturel ou du tourisme mais en tant qu'objets ethnographiques. 
Un mot sur l'édition du volume qui mérite d'être mentionnée : le beau papier, la mise en page aérée et le large format en rendent la lecture agréable. Une carte schématique (p. 12) situe les principaux groupes ethniques de la région du Mahakam. La bibliographie exhaustive sera particulièrement utile au chercheur comme au muséographe travaillant sur l'art et la culture matérielle de Bornéo.

\section{Antonio Guerreiro}

Maria Stanyukovich (ed.), Pilipinas Muna! Filippiny prejdie sjevo! K 80-ljetiou G.Y. Ratchkova (Pilipinas Muna! Les Philippines d'abord! En honneur du 80e anniversaire de Gennadiy Yevgenyevich Rachkov), série Maclay, vol. 4, Musée d'Anthropologie et d'Ethnologie, Académie Russe des Sciences, Saint Petersburg, 2011, 648 p., Ill., ISBN 978 5-88431-174-9

Ce volume est le fruit d'une conférence internationale pour célébrer le $80^{\mathrm{e}}$ anniversaire du linguiste Gennadiy Y. Rachkov. D'abord spécialiste de coréen, Rachkov s'est par la suite lancé dans l'étude du tagalog, puis fonda le Département de tagalog de l'École des Études Asiatiques et Africaines de l'Université de Saint Petersburg et le dirigea de 1967 à 2011. L'ouvrage rassemble des articles émanant d'experts en folklore, littérature, histoire, linguistique, anthropologie venus tant de Russie, que des Philippines et des États-Unis, ainsi que diverses bibliographies et catalogues de collections ethnographiques de Saint Petersbourg ayant rapport aux Philippines. Ce recueil, qui nous semble très intéressant, ne contient que quatre articles en anglais écrits respectivement par des Philippins et une Anglaise, aussi vaisje essayer de donner ici une idée du contenu en suivant le plan en quatre parties du recueil, afin de montrer la diversité et la richesse des études russes sur les Philippines.

\section{Les Philippines et la Russie}

Maria V. Stanyukovich dans son article «Les recherches sur les Philippines en Russie vues de Saint Petersburg » souligne l'importance de cette ville pour les études philippines. C'est de là que, à partir $\mathrm{du} \mathrm{XVIII}^{\mathrm{e}}$ siècle, sont partis tous les explorateurs russes, et les manuscrits et objets ethnographiques qu'ils ont rapportés s'y trouvent conservés, tant dans les archives qu'au Musée Ethnologique Pierre le Grand (Kunstkamera), le premier musée de Russie. Quant à la série de publications Maclay, elle tire son nom de l'anthropologue russe Nikolay N. Miklouho-Maclay qui, au XIX ${ }^{\mathrm{e}}$ siècle, voyagea en Nouvelle-Guinée et dans le Pacifique et se rendit trois fois aux Philippines, d'où il rapporta une riche collection d'objets toujours conservés dans ledit musée.

Ricardo Jose traite dans son article « La guerre russo-japonaise et les Philippines » de l'inspiration que la victoire nippone donna aux jeunes nationalistes philippins, dont le pays à peine débarrassé des Espagnols, venait d'être colonisé par les Américains.

Vladimir V. Noskov passe en revue les visites de la flotte russe à Manille du temps où l'empire russe essayait de s'ériger en force navale du Pacifique.

Mikhail Yu. Medvedev analyse les réformes récentes du Raja Muda Muedzul I Lail Tan Kiram du sultanat de Sulu, sud des Philippines, dans « Les symboles du sultanat royal de Sulu : héritage, contexte et réformes ». 